\title{
BMJ Open Household food insecurity among patients with pulmonary tuberculosis and its associated factors in South India: a cross-sectional analysis
}

\author{
Reshma Ayiraveetil (D , , Sonali Sarkar, ${ }^{1}$ Palanivel Chinnakali, ${ }^{1}$ \\ Kathiresan Jeyashree, ${ }^{2}$ Mathavaswami Vijayageetha, ${ }^{1}$ Pruthu Thekkur, ${ }^{3,4}$ \\ Subitha Lakshminarayanan, ${ }^{1}$ Selby Knudsen, ${ }^{5}$ Natasha S Hochberg, ${ }^{5,6}$ \\ C Robert Horsburgh, ${ }^{5,6,7}$ Jerrold Ellner, ${ }^{8}$ Gautam Roy ${ }^{1}$
}

To cite: Ayiraveetil R, Sarkar S, Chinnakali P, et al. Household food insecurity among patients with pulmonary tuberculosis and its associated factors in South India: a crosssectional analysis. BMJ Open 2020;10:e033798. doi:10.1136/ bmjopen-2019-033798

- Prepublication history and additional material for this paper are available online. To view these files, please visit the journal online (http://dx.doi. org/10.1136/bmjopen-2019033798).

Received 12 September 2019 Revised 01 February 2020 Accepted 04 February 2020

Check for updates

(C) Author(s) (or their employer(s)) 2020. Re-use permitted under CC BY-NC. No commercial re-use. See rights and permissions. Published by BMJ.

For numbered affiliations see end of article.

Correspondence to Dr Sonali Sarkar; sarkarsonaligh@gmail.com

\section{ABSTRACT}

Objectives Food insecurity is 'the limited or uncertain availability of nutritionally adequate, safe foods or inability to acquire foods in socially acceptable ways'. Majority of tuberculosis (TB) cases of resource-poor settings experience food insecurity, which impacts treatment adherence and outcomes. We aimed to determine level of household food insecurity (HFI) and its associated factors in patients with pulmonary TB.

Design This is a cross-sectional analysis of data from an ongoing cohort study.

Setting National Tuberculosis Programme (NTP) in three districts of South India.

Participants All newly diagnosed pulmonary TB cases of the cohort enrolled in the NTP at the Designated Microscopy Centres (DMCs) and Primary Health Centres (PHCs) from October 2015 to October 2018.

Primary outcome measures The proportion of baseline HFI assessed using a validated HFI Access Scale was summarised as percentage with $95 \% \mathrm{Cl}$. Possible association of sociodemographic, morbidity and behavioural characteristics with HFI was assessed using $\chi^{2}$ test, and unadjusted prevalence ratios with $95 \% \mathrm{Cl}$ were calculated. The characteristics with values of $p<0.2$ in the univariate model were included in the multivariable generalised linear model (binomial function, log link) to derive adjusted prevalence ratios (aPRs) with $95 \% \mathrm{Cl}$.

Result Of a total of 765 patients, 261 had HFI and the proportion was $34.1 \%$ (95\% Cl $30.8 \%$ to $37.6 \%$ ). Mild, moderate and severe food insecurity was found in 17 (2.2\%), 67 (8.8\%) and 177 (23.1\%) TB cases, respectively. Patients with TB who had monthly family income less than rupees 3000 (aPR 2.0; 95\% Cl 1.3 to 3.0), Karnofsky Score of 60 or less (aPR 1.5; 95\% $\mathrm{Cl} 1.1$ to 1.9 ) and those who were employed (aPR 1.4; $95 \% \mathrm{Cl} 1.0$ to 2.0) were independently associated with $\mathrm{HFI}$.

Conclusions A high level of food insecurity was seen in households with TB cases. Additional food or cash assistance for this subgroup might improve food insecurity and thereby nutritional status.

\section{Strengths and limitations of this study}

- Use of a validated tool for assessing household food insecurity (HFI) which allows cross-country comparisons.

- We used data from a prospective cohort study which implemented quality assurance checks for data collection, entry and completeness that would have reduced missing data and data errors.

- We did not study the subgroup of previously treated patients with TB in whom levels of food insecurity could be higher due to financial loss caused by repeated episodes of TB.

- The study participants were from three selected districts in South India, so generalisability of the findings is limited.

- The model developed for assessing the factors associated with HFI was deficient due to the small sample size and unavailability of a few important confounding variables.

\section{INTRODUCTION}

Tuberculosis (TB) is the leading cause of death from a single infectious agent, ranking above HIV/AIDS, and is overall the ninth leading cause of death worldwide. In 2017, there were an estimated 1.3 million TB deaths among HIV-negative people. India contributes roughly $25 \%$ of global incident TB cases and there were an estimated 421000 deaths annually due to TB in the year $2017 .{ }^{12}$ In 2014, WHO endorsed the 'End TB strategy' in line with the Sustainable Development Goals developed by the United Nations (Goals 1, 2 and 3 deal with action on poverty, hunger and ensuring healthy lives and well-being of people) with a common aim to end the global TB epidemic. ${ }^{13}$

Food security is a state in which 'all people at all times have both physical and economic 
access to sufficient food to meet their dietary needs for a productive and healthy life'. ${ }^{4}$ Catastrophic health expenditure is a common consequence of TB diagnosis, treatment and care which can lead to impoverishment and in turn food insecurity for patients with TB. Food insecurity and undernutrition share a bidirectional relationship with TB; both cause TB and could be consequences of TB. Undernutrition in patients with active TB can lead to worsening of disease, drug toxicity, drug malabsorption, and death or relapse of disease. ${ }^{5-7}$

A recent national survey (2016) in Vietnam reported that $22 \%$ of households experienced food insecurity during TB treatment, this proportion being as high as $40 \%$ among the poorest wealth quintiles. ${ }^{8}$ Food insecurity at the household level is common in India and is a strong risk factor for progression of latent infection to active TB in household contacts. ${ }^{9}$ TB in India affects poor families and communities disproportionately, with a fourfold higher prevalence in those with a low standard of living index compared with those with a high standard of living index. ${ }^{5}$ Food insecurity is also of greatest significance in households where levels of food insecurity and undernutrition are high at the time of diagnosis. Since food insecurity and undernutrition can coexist, patients with TB are unable to regain normal weight, despite effective treatment.

WHO (2013) in its guidelines 'Nutritional care and support for patients with tuberculosis' recommends assessment of food insecurity among TB cases and addressing the same with suitable packages including food assistance. ${ }^{3}$ Recently, the Government of India has launched a cash assistance scheme for all TB cases to mitigate costs and improve nutritional status. ${ }^{10}$ However, there may be households with more food insecurity that need more food assistance rather than equal assistance to all. In India, studies assessing household food insecurity (HFI) among TB cases are limited. Therefore, we aimed to determine the level of food insecurity and its associated factors using secondary data from a cohort of patients with pulmonary TB in South India.

\section{METHODS}

\section{Study design}

This is a cross-sectional analysis of data from a cohort study under Regional Prospective Observational Research for Tuberculosis (RePORT) India Consortium. Details of the study design have been previously reported. ${ }^{11-14}$

\section{National Tuberculosis Programme}

The study covers Puducherry district of the Union Territory of Puducherry (population $\sim 1.3$ million) and two adjoining districts of Tamil Nadu, that is, Villupuram (population $\sim 3.5$ million) and Cuddalore (population 2.6 million). Under the National Tuberculosis Programme (NTP), TB diagnostic and treatment services are delivered through the designated microscopy centres (DMCs) and peripheral health institutions (PHIs) under tuberculosis units as nodal points for TB control activities at subdistrict level. Sputum smear microscopy remains the central component of TB diagnosis. Under NTP, both diagnosis and treatment are provided free of cost to the patients with $\mathrm{TB}$. On diagnosis of TB, the patients are referred to the nearest PHI for initiation of treatment. Morbidity details (diabetes, HIV) and medication adherence, follow-up details and TB treatment outcomes of these patients are documented in individual TB treatment cards.

\section{RePORT International}

RePORT International represents a consortium of regional cohorts (RePORT India, RePORT Brazil, RePORT South Africa, RePORT China, RePORT Philippines and RePORT Indonesia) that are linked through the implementation of a common protocol for data and specimen collection. The objectives and composition of RePORT International are described elsewhere. ${ }^{15}$

One of the five teams under RePORT India, the Jawaharlal Institute of Postgraduate Medical Education and Research (JIPMER), Boston Medical Centre and Rutgers University, has established a pulmonary TB cohort of adults and children $\geq 6$ years and their household contacts to identify biomarkers for risk of TB treatment failure and risk of development of TB in household contacts. TB cases diagnosed under NTP in the three districts (Puducherry, Cuddalore and Villupuram) were enrolled in the cohort at the DMCs and public health centres since 2014. Only newly diagnosed smear-positive and culture-positive pulmonary TB cases were included. Details on data collection and procedures were previously reported. ${ }^{11-14}$

\section{Study population}

For this analysis, we included all TB cases in the cohort enrolled from October 2015 to October 2018. Written informed consent was obtained from all participants before enrolment. Multidrug resistant and extremely drug resistant $\mathrm{TB}$ cases at diagnosis were excluded.

\section{Study tool}

The Household Food Insecurity Access Scale (HFIAS) ${ }^{4}$ was used to assess food insecurity in the households in the past 30 days. The scale consists of nine items grouped under three domains: (1) Anxiety or uncertainty about the household food supply. (2) Insufficient quality. (3) Insufficient food intake and it's physical consequences. ${ }^{4}$ The respondent is first asked an occurrence question, whether the condition in the question happened at all in the past 4 weeks (yes or no). If the respondent answers 'yes' to an occurrence question, a frequency-ofoccurrence question is asked to determine whether the condition happened rarely (1-2 times), sometimes (3-10 times) or often (more than 10 times) in the past 4 weeks. Each item is scored on a range of 0 to 3; 0 for 'no occurrence' and 3 for 'often'. The minimum and maximum scores for a household are 0 and 27, respectively. The 
scores were categorised into four levels of HFI: food secure and mild, moderately and severely food insecure ${ }^{4}$ online supplementary file 1 .

The Alcohol Use Disorders Identification Test (AUDIT)-C Questionnaire (a modified version of AUDIT $)^{16}$ was used to assess alcohol use among participants.

\section{Data extraction, analysis and statistics}

Of a total of 1229 TB cases enrolled in the cohort, we extracted data for 765 cases excluding two childhood TB cases; the initial 462 cases enrolled were not assessed for HFI because of not having the HFIAS in the study proforma during the initial phase of the project. The HFIAS was introduced in the revised study proforma after the 462 patients were already enrolled in the project. However, there was no difference in the baseline sociodemographic and clinical characteristics of the 462 patients excluded, compared with those included in the study.

Data were extracted from the RePORT India project database for the JIPMER site in a deidentified manner and analysed using Stata V.12.0 software. The proportion of HFI was summarised as percentage with $95 \%$ CI. Possible association of sociodemographic, morbidity-related and behavioural characteristics with HFI was assessed using $\chi^{2}$ test, and unadjusted prevalence ratios with $95 \%$ CI were calculated. The characteristics with a value of $p<0.2$ in the univariate model were included in the multivariable generalised linear model (binomial function and log link) to derive adjusted prevalence ratios (aPRs) with 95\% CI. The variables such as marital status, education, residence, number of earners in the household, HIV status, tobacco use, and alcohol use were not included for multivariate analysis.

\section{Patient and public involvement}

There was no patient or public involvement.

\section{RESULTS}

The mean (SD) age of the 765 individuals included in analysis was $44^{14}$ years; $611(80 \%)$ were male. Sociodemographic characteristics of the TB cases are described in table 1. Of the total participants, 131 (17\%) did not have any formal education, about $77 \%$ were employed and $11 \%$ had monthly family income less than rupees 3000 ( $\sim$ US\$43). Behavioural and disease-related characteristics are described in table 2. More than half $(58 \%)$ were alcohol users (in the previous year) and 30\% were current tobacco users. Of the total, $470(61 \%)$ were underweight (body mass index (BMI) $<18.5 \mathrm{~kg} / \mathrm{m} 2$ ) and 5 patients $(0.6 \%)$ were HIV-infected. The Karnofsky Score was 60 or less (require assistance for routine activities) in $29 \%$ of patients.

Overall, 261 patients had HFI and the proportion was $34.1 \%$ (95\% CI $30.8 \%$ to $37.6 \%$ ). Mild, moderate and severe food insecurity was found in 17 (2.2\%), 67 (8.8\%) and $177(23.1 \%)$ TB cases, respectively. Components of
Table 1 Sociodemographic characteristics of individuals with pulmonary tuberculosis (TB) in three districts of South India, 2015-2018 $(n=765)$

\begin{tabular}{|c|c|}
\hline Characteristics & Frequency $(\%)$ \\
\hline \multicolumn{2}{|l|}{ Age (in years) } \\
\hline $15-29$ & $130(17.0)$ \\
\hline $30-44$ & $229(29.9)$ \\
\hline $45-59$ & $292(38.2)$ \\
\hline 60 and above & $114(14.9)$ \\
\hline \multicolumn{2}{|l|}{ Gender } \\
\hline Male & $611(79.9)$ \\
\hline Female & $154(20.1)$ \\
\hline \multicolumn{2}{|l|}{ Marital status } \\
\hline Never married & $132(17.3)$ \\
\hline Married/living together & $567(74.1)$ \\
\hline Separated/divorced/ widowed & $66(8.6)$ \\
\hline \multicolumn{2}{|l|}{ Education (years of schooling) } \\
\hline No formal education & $131(17.1)$ \\
\hline $1-5$ & $160(20.9)$ \\
\hline $6-10$ & $317(41.4)$ \\
\hline$>10$ & $157(20.5)$ \\
\hline \multicolumn{2}{|l|}{ Employment } \\
\hline Employed & $588(76.9)$ \\
\hline Unemployed & $177(23.1)$ \\
\hline \multicolumn{2}{|c|}{ Household Income per month (in rupees) } \\
\hline$<3000$ & $80(10.5)$ \\
\hline $3000-5000$ & $296(38.7)$ \\
\hline $5001-10000$ & $279(36.5)$ \\
\hline$>10000$ & $94(12.3)$ \\
\hline Didn't answer & $16(2.0)$ \\
\hline \multicolumn{2}{|l|}{ Number of individuals in house } \\
\hline$\leq 3$ & $604(78.9)$ \\
\hline$>3$ & $161(21.1)$ \\
\hline \multicolumn{2}{|l|}{ Residence* } \\
\hline Urban & $338(44.2)$ \\
\hline Rural & $409(53.4)$ \\
\hline Not recorded & $18(2.4)$ \\
\hline \multicolumn{2}{|l|}{ Number of earners in the household } \\
\hline None & $15(1.9)$ \\
\hline One & $509(66.5)$ \\
\hline Two or more & $241(31.6)$ \\
\hline \multicolumn{2}{|l|}{ Religion } \\
\hline Hindu & $677(88.5)$ \\
\hline Christianity & $54(7.1)$ \\
\hline Muslim & $32(4.2)$ \\
\hline Others & $2(0.3)$ \\
\hline
\end{tabular}

food insecurity are described in table 3 . Worry or anxiety about not having enough food was reported in $15 \%$ of TB households. In $21 \%$ of TB households, eating fewer meals in a day due to lack of enough food was reported. 
Table 2 Morbidity and behavioural characteristics of individuals with pulmonary tuberculosis (TB) in three districts of South India, 2015-2018 $(n=765)$

\begin{tabular}{|c|c|}
\hline Characteristic & Frequency $(\%)$ \\
\hline \multicolumn{2}{|c|}{ Sputum smear grading at diagnosis } \\
\hline $1+$ & $241(31.5)$ \\
\hline $2+$ & $255(33.3)$ \\
\hline $3+$ & $269(35.2)$ \\
\hline \multicolumn{2}{|c|}{ Karnofsky Score at diagnosis } \\
\hline $50-60$ & $218(28.5)$ \\
\hline$>60$ & $547(71.5)$ \\
\hline \multicolumn{2}{|l|}{ HIV status } \\
\hline Seropositive & $5(0.6)$ \\
\hline Seronegative & $760(99.4)$ \\
\hline \multicolumn{2}{|l|}{ RBS } \\
\hline$<200 \mathrm{mg} / \mathrm{dL}$ & $531(69.4)$ \\
\hline$\geq 200 \mathrm{mg} / \mathrm{dL}$ & $234(30.6)$ \\
\hline \multicolumn{2}{|c|}{ Any other comorbidity* } \\
\hline Yes & $154(20.2)$ \\
\hline No & $611(79.8)$ \\
\hline \multicolumn{2}{|l|}{ BMI } \\
\hline$<18.5$ & $470(61.4)$ \\
\hline $18.5-22.9$ & $221(28.9)$ \\
\hline $23-24.9$ & $42(5.5)$ \\
\hline 25 and above & $29(3.8)$ \\
\hline Not recorded & $3(0.4)$ \\
\hline \multicolumn{2}{|l|}{ Alcohol use† } \\
\hline Ever & $446(58.3)$ \\
\hline Never & $319(41.7)$ \\
\hline \multicolumn{2}{|l|}{ Tobacco use } \\
\hline Former & $140(18.3)$ \\
\hline Current & $231(30.2)$ \\
\hline Never & $394(51.5)$ \\
\hline
\end{tabular}

*Other comorbidities such as asthma, hepatitis, renal disease, cancer and breathing difficulty were reported by the participants.

†Alcohol use was measured for the past 1 year.

‡Current or prior habitual use of both smoking and smokeless forms of tobacco.

$\mathrm{BMI}$, body mass index; RBS, random blood sugar.

Level of food insecurity in different subgroups is presented in table 4 . In adjusted analysis, TB cases who had monthly family income less than rupees 3000 (aPR 2.0; 95\% CI 1.3 to 3.0), Karnofsky Score of 60 or less (aPR $1.5 ; 95 \%$ CI 1.1 to 1.9 ) and those who were employed (aPR 1.4; 95\% CI 1.0 to 2.0) had higher proportion of HFI.

\section{DISCUSSION}

Our study of newly diagnosed patients with pulmonary TB in the public sector in South India revealed that about a third of patients with TB experienced HFI and about one out of four patients experienced severe food insecurity at the time of diagnosis. Level of food insecurity was high in the low-income groups, those employed and those who had severe illness.

Prevalence of food insecurity in the general population of India is also high ranging from $45.5 \%$ to $77.2 \% .^{17} 18$ Hence, HFI among patients with TB is common as it can be both a cause and consequence of TB. The national level survey from Vietnam (2016) reported 22\% of patients with TB experienced HFI; lower levels of $6 \%$ were reported among patients with TB in Sri Lanka. ${ }^{8} 19$

Food insecurity was twice as high in low-income TB households (< rupees 3000) compared with their higher income counterparts. Catastrophic health expenditure, a consequence of TB diagnosis and treatment, can lead to worsening of food insecurity in low-income groups during the course of the disease. ${ }^{5}$ These subgroups need to be provided additional assistance instead of 'equal for all' food or cash assistance benefits. Since income is usually under-reported, identifying such target groups may not be an easy task.

Food insecurity at the household level is a strong risk factor for progression of latent infection to active TB in household contacts. ${ }^{9}{ }^{20}$ Since food insecurity measures are applicable to all households, a wider approach of reducing food insecurity targeting all household contacts is needed.

In our study, about $60 \%$ of the patients with TB were underweight. Undernutrition is both an important risk factor for, and a common consequence of, TB. In food insecure households, undernutrition could be an intermediary step in the nutritional pathway of food insecurity leading to morbidity like TB. In India, undernutrition is highly prevalent in patients with $\mathrm{TB}$ and the dietary intake of calories is significantly lower $(500-700$ calories) than recommended. ${ }^{21}$ As recommended by WHO, addressing undernutrition through nutritional counselling and support should be considered as part of the standard of care for people with TB. The recently launched 'Nikshay Poshan Yojana', a direct benefit transfer scheme by the government of India is a welcome step towards addressing undernutrition. ${ }^{22}$ Our study did not include severely ill patients (Karnofsky Score <40) and previously treated patients with $\mathrm{TB}$ in whom undernutrition rates are expected to be high. This may partly explain why our study did not find an association between food insecurity and undernutrition, though previous studies have reported otherwise. ${ }^{23-26}$ Also, we have assessed food insecurity at the household level, and BMI assessed is that of the individual patients. May be the patient's nutrition is maintained at the expense of other family members, so he or she may have had normal BMI. However, the temporality of the BMI, HFI and weight loss could not be established due to the cross-sectional nature of this study and we also failed to account for the sequence of these events during analysis. Thus, we fail to strongly comment on the causal pathways of association between HFI, BMI and weight loss. Also, we couldn't explore details on 


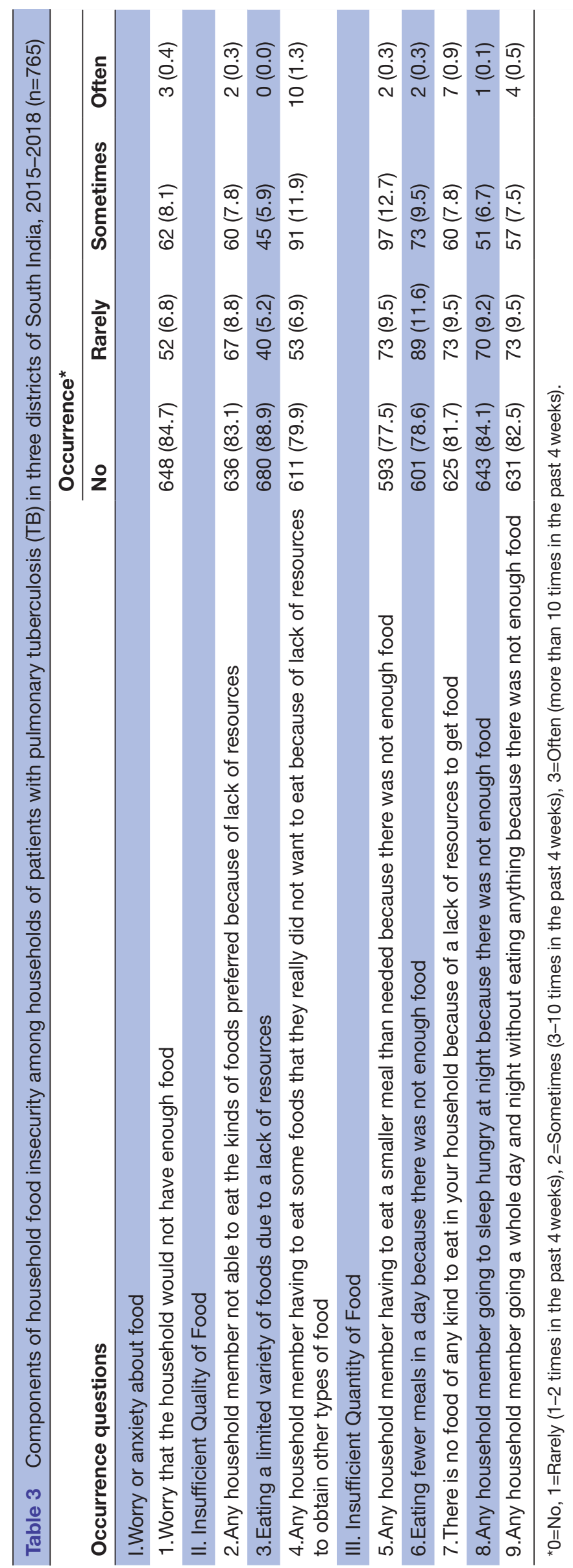


Table 4 Association of sociodemographic, morbidity and behavioural characteristics with household food insecurity among individuals with pulmonary TB in Puducherry, $n=765$

\begin{tabular}{|c|c|c|c|c|}
\hline Characteristics & Total & Food insecurity* & $\begin{array}{l}\text { Unadjusted PR† } \\
(95 \% \mathrm{Cl}) \neq\end{array}$ & $\begin{array}{l}\text { Adjusted PR†, § } \\
(95 \% \mathrm{Cl}) \ddagger\end{array}$ \\
\hline Total & 765 & $261(34.1)$ & - & - \\
\hline \multicolumn{5}{|l|}{ Age (in years) } \\
\hline $15-29$ & 130 & 48 (36.9) & $1.4(0.9-2.0)$ & $1.2(0.7-2.2)$ \\
\hline $30-44$ & 229 & $86(37.6)$ & $1.4(1.0-1.9)$ & $1.4(0.9-2.2)$ \\
\hline $45-59$ & 292 & $96(39.9)$ & $1.2(0.9-1.7)$ & $1.2(0.8-1.8)$ \\
\hline 60 and above & 114 & $31(27.2)$ & 1.0 & Ref \\
\hline \multicolumn{5}{|l|}{ Gender } \\
\hline Male & 611 & 203 (33.2) & 1.0 & Ref \\
\hline Female & 154 & $58(37.7)$ & $1.1(0.91 .4)$ & $1.2(0.8-1.8)$ \\
\hline \multicolumn{5}{|l|}{ Marital status } \\
\hline Never married & 132 & $42(31.8)$ & $0.9(0.7-1.2)$ & - \\
\hline Married/living together & 567 & $196(34.6)$ & 1.0 & - \\
\hline Separated/divorced/widowed & 66 & $23(34.9)$ & $1.008(0.7-1.4)$ & - \\
\hline \multicolumn{5}{|l|}{ Education (years of schooling) } \\
\hline No formal education & 131 & $40(30.5)$ & 1.0 & - \\
\hline $1-5$ & 160 & $54(33.7)$ & $1.10(0.8-1.5)$ & - \\
\hline $6-10$ & 317 & $114(34.0)$ & $1.2(0.9-1.6)$ & - \\
\hline$>10$ & 157 & $53(33.8)$ & $1.1(0.8-1.6)$ & - \\
\hline \multicolumn{5}{|l|}{ Employment } \\
\hline Employed & 588 & $206(35.0)$ & $1.1(0.9-1.4)$ & $1.4(1.0-2.0)$ \\
\hline Unemployed & 177 & $55(31.7)$ & 1.0 & Ref \\
\hline \multicolumn{5}{|c|}{ Household income per month (in rupees) } \\
\hline$<3000$ & 80 & $41(51.3)$ & $1.9(1.3-2.4)$ & $2.0(1.3-3.0)$ \\
\hline $3000-5000$ & 296 & $107(36.2)$ & $1.3(1.0-1.6)$ & $1.3(0.9-1.7)$ \\
\hline $5001-10000$ & 279 & $80(28.7)$ & 1.0 & Ref \\
\hline$>10000$ & 94 & $24(25.5)$ & $0.9(0.6-1.3)$ & $0.9(0.6-1.6)$ \\
\hline Didn't answer & 16 & $9(56.3)$ & $2.0(1.2-3.1)$ & $2.2(1.1-4.5)$ \\
\hline \multicolumn{5}{|l|}{ Number of individuals in the house } \\
\hline$\leq 3$ & 604 & $198(32.8)$ & 1.0 & Ref \\
\hline$>3$ & 161 & $63(39.1)$ & $1.2(0.9-1.5)$ & $1.3(0.9-1.8)$ \\
\hline \multicolumn{5}{|l|}{ Residenceी } \\
\hline Urban & 338 & $114(33.7)$ & 1.0 & - \\
\hline Rural & 409 & $143(34.0)$ & $1.03(0.8-1.3)$ & - \\
\hline \multicolumn{5}{|c|}{ Number of earners in the household } \\
\hline None & 15 & 5 (33.3) & 1.0 & - \\
\hline One & 509 & $177(34.8)$ & $1.04(0.5-2.2)$ & - \\
\hline Two or more & 241 & $79(32.8)$ & $0.9(0.5-2.1)$ & - \\
\hline \multicolumn{5}{|l|}{ Sputum smear grading at diagnosis } \\
\hline $1+$ & 241 & $68(28.2)$ & 1.0 & Ref \\
\hline $2+$ & 255 & $93(36.5)$ & $1.3(1.0-1.7)$ & $1.3(0.9-1.7)$ \\
\hline $3+$ & 269 & $100(37.2)$ & $1.3(1.0-1.7)$ & $0.4(0.1-1.3)$ \\
\hline \multicolumn{5}{|l|}{ Karnofsky Score at diagnosis } \\
\hline $50-60$ & 218 & $97(44.5)$ & $1.5(1.2-1.8)$ & $1.5(1.1-1.9)$ \\
\hline
\end{tabular}


Table 4 Continued

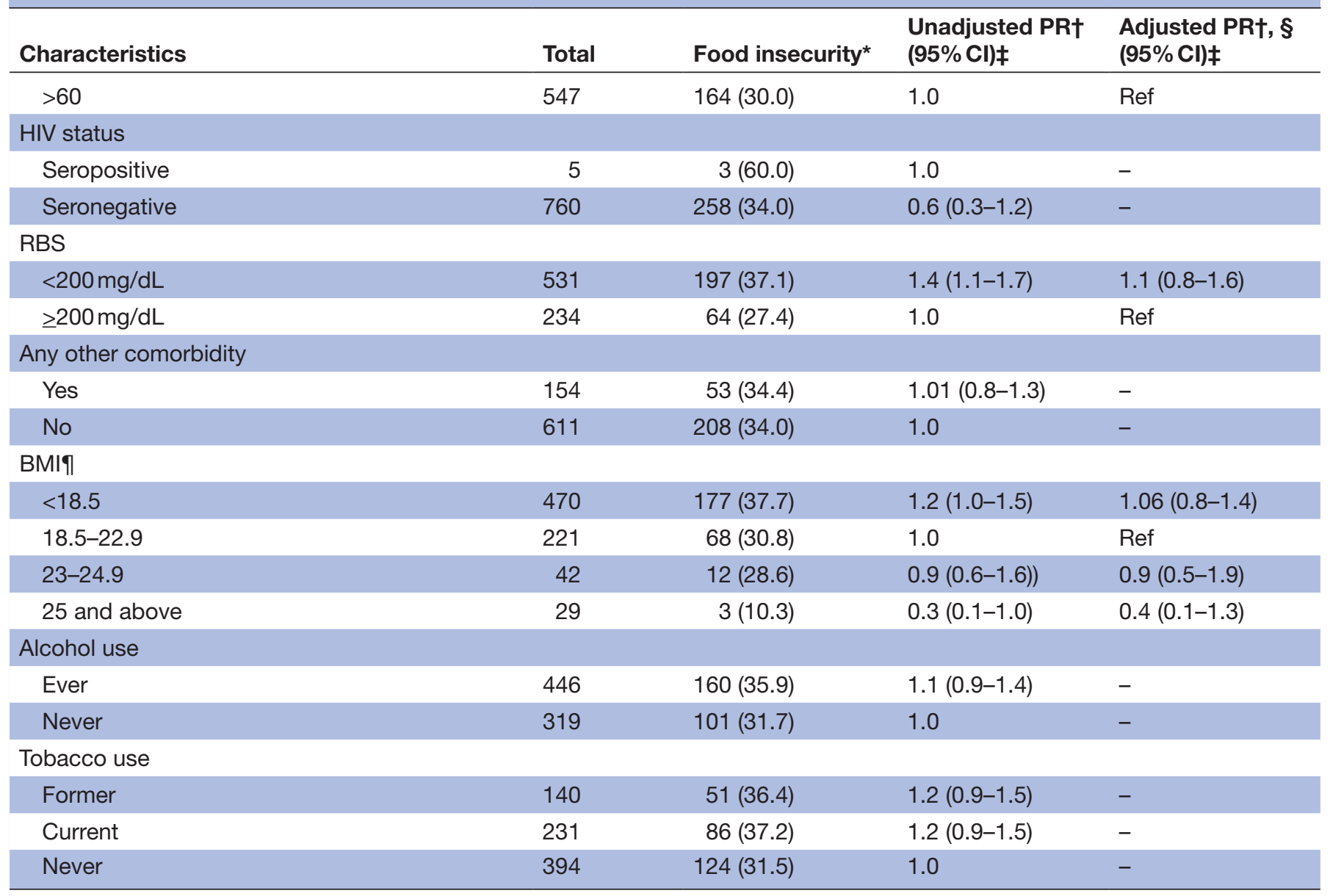

*Level of pood insecurity was assessed using the Household Food Insecurity Assessment Scale (HFIAS) for Measurement of Food AccessFANTAIII.

†PR, prevalence ratio.

$\ddagger \mathrm{Cl}$.

$\S$ Adjusted for characteristics with a value of $p<0.2$ in the univariate model.

ПResidence-data are missing for 18 participants; Body Mass Index-data are missing for 3 participants.

$\mathrm{BMI}$, body mass index; RBS, random blood sugar; TB, tuberculosis.

employment such as type of employment, which could be a risk factor for development of TB and could have influenced the income and thus the ability to purchase food items also.

Several studies support the notion that food insecurity negatively affects treatment adherence. Conditions of food insecurity (lack of adequate food, concern about daily food production) contribute to non-adherence to $\mathrm{TB}$ treatment as reported by qualitative studies. ${ }^{27-29}$ Hence, identifying food insecurity at the time of diagnosis and linking the patients to food assistance or social security programmes is needed. We plan to report the effect of food insecurity on adherence and TB treatment outcomes in a separate paper.

Strengths of the study include use of a validated tool ${ }^{4}$ for assessing HFI which allows cross-country comparisons. We used the data from a prospective cohort study which implemented quality assurance checks for data collection and entry that would have reduced missing data and data errors. There were a few limitations in the study. Our study included patients identified in the public sector alone and food insecurity levels may be different in patients accessing $\mathrm{TB}$ care in the private sector. Since, repeated episodes of TB may be a cause for financial loss leading to food insecurity, the levels could be higher in previously treated patients with TB and we did not study this subgroup. The study participants were from three selected districts in South India, so generalisability of the findings is limited. Being a cross-sectional analysis, causal relationships of factors with food insecurity cannot be inferred. The model we constructed for exploring factors associated with HFI was deficient as a few important confounding variables were included and also, the small sample size (power) was less for performing rational statistical analyses. Thus, the factors associated with HFI need to be interpreted with caution considering this major limitation in the multivariate analysis. 


\section{CONCLUSION}

To conclude, HFI was experienced by one in three patients with TB and this was twice more in low-income groups. Additional food or cash assistance to food insecure patients with $\mathrm{TB}$ and household contacts will improve food insecurity and undernutrition.

\section{Author affiliations}

${ }^{1}$ Department of Preventive and Social Medicine, Jawaharlal Institute of

Postgraduate Medical Education and Research (JIPMER), Puducherry, India

${ }^{2}$ Department of Community Medicine, Velammal Medical College, Madurai,

Tamilnadu, India

${ }^{3}$ Centre for Operational Research, International Union Against Tuberculosis and Lung Disease, Paris, France

${ }^{4}$ The Union South-East Asia Office, New Delhi, India

${ }^{5}$ Section of Infectious Diseases, Department of Medicine, Boston University School of Medicine, Massachusetts, United States

${ }^{6}$ Department of Epidemiology, Boston University School of Public Health, Massachusetts, United States

${ }^{7}$ Department of Global Health, Boston University School of Public Health, Massachusetts, United States

${ }^{8}$ Department of Medicine, Rutgers University, New Jersey, United States

Acknowledgements This cross-sectional analysis was conducted through the Structured Operational Research and Training Initiative (SORT IT), a global partnership led by the Special Programme for Research and Training in Tropical Diseases at the World Health Organisation (WHO/TDR). The model is based on a course developed jointly by the International Union Against Tuberculosis and Lung Disease (The Union) and Medécins sans Frontières (MSF/Doctors Without Borders). The specific SORT IT programme which resulted in this publication was jointly developed and implemented by: The Union South-East Asia Office, New Delhi, India; the Centre for Operational Research, The Union, Paris, France; Department of Preventive and Social Medicine, Jawaharlal Institute of Postgraduate Medical Education and Research, Puducherry, India; Department of Community Medicine and School of Public Health, Postgraduate Institute of Medical Education and Research, Chandigarh, India; Department of Community Medicine, All India Institute of Medical Sciences, Nagpur, India; Dr. Rajendra Prasad Centre for Ophthalmic Sciences, All India Institute of Medical Sciences, New Delhi, India; Department of Community Medicine, Pondicherry Institute of Medical Science, Puducherry, India; Department of Community Medicine, Kalpana Chawla Medical College, Karnal, India; National Centre of Excellence and Advance Research on Anaemia Control, All India Institute of Medical Sciences, New Delhi, India; Department of Community Medicine, Sri Manakula Vinayagar Medical College and Hospital, Puducherry, India; Department of Community Medicine, Velammal Medical College Hospital and Research Institute, Madurai, India; Department of Community Medicine, Yenepoya Medical College, Mangalore, India; Karuna Trust, Bangalore, India and National Institute for Research in Tuberculosis, Chennai, India. Authors would like to thank the funders, the index cases, their families, and the staff that worked on the RePORT India project. We also acknowledge the contribution of the Data Coordinating Centre at Boston Medical Centre, US.

Contributors RA:Principal Investigator (PI), conception/ design of the protocol, data capture, development of data capture tool, data analysis/interpretation, drafting/critically reviewing the paper, giving approval for the final version to be published. SS: Acquisition of data, conception/ design of the protocol, revision of manuscript, critically reviewing the paper, giving approval for the final version to be published. PC: Conception/design of the protocol, development of data capture tool, data analysis/interpretation, drafting/critically reviewing the paper, giving approval for the final version to be published. KJ: Conception/design of the protocol, development of data capture tool, data analysis/interpretation, drafting/critically reviewing the paper, giving approval for the final version to be published. MV: Data analysis and interpretation, critically reviewing the paper, giving approval for the final version to be published. PT: Conception, data analysis, revision of manuscript, critically reviewing the paper, giving approval for the final version to be published. $\mathrm{SL}$ : Conception, project management, critically reviewing the manuscript, giving approval for the final version to be published. SK: Data curation and management, reviewing the manuscript, giving approval for the final version to be published. NSH: Project administration and supervision, critically reviewing and editing the manuscript, giving approval for the final version to be published. CRH: Critically reviewing and editing the manuscript, giving approval for the final version to be published. JE: Acquisition of data, critically reviewing the manuscript, giving approval for the final version to be published. GR: Acquisition of data, critically reviewing the manuscript, giving approval for the final version to be published.

Funding The training programme, within which this paper was developed, and the open access publication costs were funded by the Department for International Development (DFID), UK and La Fondation Veuve Emile Metz-Tesch (Luxembourg). The RePORT India project has been funded in whole or in part with Federal funds from the Government of India's (GOI) Department of Biotechnology (DBT), the Indian Council of Medical Research (ICMR), the US National Institutes of Health (NIH), National Institute of Allergy and Infectious Diseases (NIAID), Office of AIDS Research (OAR), and distributed in part by Civilian Research and Development Foundation (CRDF) Global.

Disclaimer The contents of this publication are solely the responsibility of the authors and do not represent the official views of the DBT, the ICMR, the NIH, or CRDF Global. The funders had no role in study design, data collection and analysis, decision to publish, or preparation of the manuscript.

Competing interests None declared.

Patient consent for publication Not required.

Ethics approval Ethics approval was obtained from the Institutional Ethics Committee of JIPMER (Ref.No: JIP/IEC/2013/4/194) and Institute Review Board of Boston Medical Center (IRB No: H-32657/7-05-2017) for the cohort study. The study protocol for this secondary analysis was reviewed and approved by the Ethics Advisory Group of the International Union Against Tuberculosis and Lung Disease (99/18), Paris, France.

Provenance and peer review Not commissioned; externally peer reviewed.

Data availability statement Data are available upon reasonable request. The technical appendix, statistical code and data set will be available upon request from the Corresponding author.

Open access This is an open access article distributed in accordance with the Creative Commons Attribution Non Commercial (CC BY-NC 4.0) license, which permits others to distribute, remix, adapt, build upon this work non-commercially, and license their derivative works on different terms, provided the original work is properly cited, appropriate credit is given, any changes made indicated, and the use is non-commercial. See: http://creativecommons.org/licenses/by-nc/4.0/.

\section{ORCID iD}

Reshma Ayiraveetil http://orcid.org/0000-0002-2417-6639

\section{REFERENCES}

1 World Health Organization. End TB global tuberculosis report 2017. Geneva, 2017: 147.

2 Central TB Division. TB India-Annual Report 2017. India TB Rep [Internet]. 2017;1-173. Available: https://tbcindia.gov.in/ WriteReadData/TB India 2017.pdf

3 World Health Organization. WHO Guideline : Nutritional care and support for patient with tuberculosis. In: WHO Guidel Nutr care support patient with Tuberc. World Heal Organ, 2013.

4 Coates J, Swindale A, Bilinsky P, et al. Household food insecurity access scale (HFIAS) for measurement of food access: indicator guide. Washington, DC: Food Nutr Tech, 2007.

5 Central TB Division. Guidance document: nutritional care and support for patients with tuberculosis in India, 2017. Available: http:// www.tbcindia.nic.in/WriteReadData/Guidance Document - Nutritional Care \%26 Support for TB patients in India.pdf

6 Bhargava A, Benedetti A, Oxlade O, et al. Undernutrition and the incidence of tuberculosis in India: national and subnational estimates of the population-attributable fraction related to undernutrition. Natl Med J India 2014;27:128-33.

7 Sinha P, Davis J, Saag L, et al. Undernutrition and tuberculosis: public health implications. J Infect Dis 2019;219:1356-63.

8 Nhung NV, Hoa NB, Anh NT, et al. Measuring catastrophic costs due to tuberculosis in Viet Nam. Int $J$ Tuberc Lung Dis 2018;22:983-90.

9 Jubulis J, Kinikar A, Ithape M, et al. Modifiable risk factors associated with tuberculosis disease in children in Pune, India. Int J Tuberc Lung Dis 2014;18:198-204.

10 Central TB Division. National strategic plan for tuberculosis elimination 2017-2025. Dir Gen Heal Serv Minist Heal Fam Welf 2017:110-108. 
11 Van Ness SE, Chandra A, Sarkar S, et al. Predictors of delayed care seeking for tuberculosis in southern India: an observational study. BMC Infect Dis 2017;17:1-9.

12 Hochberg NS, Sarkar S, Horsburgh CR, et al. Comorbidities in pulmonary tuberculosis cases in Puducherry and Tamil Nadu, India: opportunities for intervention. PLoS One 2017;12:e0183195.

13 Hoyt KJ, Sarkar S, White L, et al. Effect of malnutrition on radiographic findings and mycobacterial burden in pulmonary tuberculosis. PLoS One 2019;14:e0214011-11.

14 Leong S, Zhao Y, Joseph NM, et al. Existing blood transcriptional classifiers accurately discriminate active tuberculosis from latent infection in individuals from South India. Tuberculosis 2017;2018:41-51.

15 Hamilton CD, Swaminathan S, Christopher DJ, et al. Report international: advancing tuberculosis biomarker research through global collaboration. Clin Infect Dis 2015;61:S155-9.

16 Babor TF, Saunders JCH-BJB, Monteiro MG. The alcohol use disorder identification test. guidelines for use in primary care. World Heal Organ Geneva 2015;2:1-41.

17 Chinnakali P, Upadhyay RP, Shokeen D, et al. Prevalence of household-level food insecurity and its determinants in an urban resettlement colony in North India. J Heal Popul Nutr 2014;32:227-36.

18 Joshi A, Arora A, Amadi-Mgbenka C, et al. Burden of household food insecurity in urban slum settings. PLoS One 2019;14:e0214461-24.

19 Jayasuriya NA, Nayanathara L, Iddamalgoda N, et al. Food security and nutrition among the tuberculosis infected patients. A case study among the patients screened at chest clinic of medical research Institute of Colombo, Sri Lanka. World Food Programme: Food security analysis(VAM), 2014.

20 Bloem MW, Saadeh R. Foreword: the role of nutrition and food insecurity in HIV and tuberculosis infections and the implications for interventions in resource-limited settings. Food Nutr Bull 2010;31:S289-91.

21 Swaminathan S, Padmapriyadarsini C, Sukumar B, et al. Nutritiona status of persons with HIV infection, persons with HIV infection and tuberculosis, and HIV-negative individuals from southern India. Clin Infect Dis 2008;46:946-9.

22 Yadav S, Atif M, Rawal G. Nikshay Poshan Yojana- another step to eliminate TB from India. IP Indian J Immunol Respir Med 2018;3:28-9.

23 Gupta K, Gupta R, Atreja A, et al. Tuberculosis and nutrition. Lung India 2009;26:9-16.

24 Park HO, Kim SH, Moon SH, et al. Association between body mass index and sputum culture conversion among South Korean patients with multidrug resistant tuberculosis in a tuberculosis referral hospital. Infect Chemother 2016;48:317-23.

25 Lönnroth K, Williams BG, Stadlin S, et al. Alcohol use as a risk factor for tuberculosis - a systematic review. BMC Public Health 2008;8:289.

26 Podewils LJ, Holtz T, Riekstina V, et al. Impact of malnutrition on clinical presentation, clinical course, and mortality in MDR-TB patients. Epidemiol Infect 2011;139:113-20.

27 Mekonnen HS, Azagew AW. Non-adherence to anti-tuberculosis treatment, reasons and associated factors among TB patients attending at Gondar town health centers, Northwest Ethiopia 11 medical and health sciences 1103 clinical sciences 11 medical and health sciences 1117 public Hea. BMC Res Notes 2018;11:1-8.

28 Munro SA, Lewin SA, Smith HJ, et al. Patient adherence to tuberculosis treatment: a systematic review of qualitative research. PLoS Med 2007;4:e238-45.

29 Diefenbach-Elstob T, Plummer D, Dowi R, et al. The social determinants of tuberculosis treatment adherence in a remote region of Papua New Guinea. BMC Public Health 2017;17:1-12. 